\title{
Diferencias en la traducción de términos griegos del campo léxico 'mujer' en la Biblia gótica de Úlfilas ${ }^{1}$
}

\author{
Miguel Ayerbe Linares \\ Universidad del País Vasco/Euskal Herriko Unibertsitatea \\ miguel.ayerbe@ehu.es
}

https://dx.doi.org/10.12795/futhark.2013.i08.01

\begin{abstract}
In any language, we expect a given term or expression to be used when referring to the same thing. Something similar is to be expected of a translation. However, in certain translations of religious texts featuring the Virgin Mary, this does not seem to apply. Apparently, the choice of words used for the lexical field of "woman" varies when it is referring to the Virgin Mary. This difference of usage is shown by the fact that in this type of texts, there are certain terms related to the lexical field of "woman" that are never used with reference to the Virgin Mary, whereas some others are used only with reference to this figure, and not to other women. In this paper, my aim is to show this circumstance, using fragments from the Gothic Bible, translated from Greek by the Arian bishop Ulfilas ( $4^{\text {th }}$ century). I will gather the terms that are used in gothic to refer to a woman, whether it is the Virgin Mary or not, and I will also analyse the contexts in which these terms are used, in order to discover what specific terms are used only with reference to the Virgin Mary, and in which specific contexts, and what terms are not used with reference to her. Finally, I will attempt to explain the reasons that might reasonably have given rise to these distinctions.
\end{abstract}

Key words: Biblical Translation, terminological distinctions, Virgin Mary, gothic language, lexical field, woman.

Resumen: En cualquier lengua cabe esperar que, para referentes similares, se use el mismo término o expresión. Algo parecido cabe esperar también en la traducción. Ahora bien, en la traducción de textos de carácter religioso

\footnotetext{
El nombre de este obispo arriano (310-383) puede encontrarse escrito de diversas formas, según la fuente que se consulte y la lengua en que se consulte. Además de esta forma, puede encontrarse también como Ulfila, Úlfila, Ulfilas, Wulfila, Wulfilas. Todas ellas parten del lexema (w)ulf- "lobo" al que se le añade el sufijo diminutivo -il-, resultando "lobezno".
} 
donde aparece la figura de la Virgen María, parece constatarse indicios de que esto no es así. Efectivamente, parece constatarse que no siempre se hace el mismo uso de los términos del campo léxico 'mujer' cuando el referente es precisamente la Virgen María. Esta diferencia de uso se materializa en el hecho de que, en este tipo de textos, existen términos del campo léxico 'mujer' cuyo uso queda excluido para referirse a la Virgen María, mientras otros son empleados para referirse exclusivamente a ella y no a otras mujeres. En el presente trabajo, el objetivo es poner de manifiesto esta realidad, a través de la traducción de los fragmentos de la biblia gótica, obra del obispo arriano Úlfilas (s. IV), a partir de la lengua griega. Para ello, serán recogidos los términos empleados en gótico para referirse a una mujer, sea ésta la Virgen María u otra. También serán analizados los contextos en los que dichos términos se utilizan, al objeto de constatar qué términos concretos y en qué situaciones concretas se emplean exclusivamente para la Virgen María y cuáles son evitados para referirse a ella. Finalmente, propondremos los motivos que, a nuestro entender, han podido llevar a establecer dichas distinciones.

Palabras clave: Traducción bíblica, distinción terminológica, Virgen María, lengua gótica, campo léxico, mujer.

\section{Introducción y antecedentes}

La traducción de la Biblia ha sido con frecuencia objeto de estudio, ya que desde su concepción ha sido un libro leído a lo largo de los siglos, y para ello fue necesario también traducirlo antes a muchas lenguas. Dichas traducciones, además, han sufrido muchas modificaciones a lo largo de los siglos, debido a que las diversas lenguas meta han ido evolucionando, tanto en su estructura morfológica y sintáctica, como en las relaciones significante-significado-referente. Por este motivo, la Biblia constituye un interesante objeto de estudio, tanto en la historia de la traducción como en la traducción en sí misma. Concretamente en el ámbito léxico-semántico, la Biblia ofrece un campo de estudio especialmente atractivo, pues constituye un importante vehículo para transmitir la vida de Dios hecho hombre y sus enseñanzas, lo cual exigía un riguroso cuidado en la exposición y narración de su contenido, al objeto de evitar desviaciones del mensaje que Dios quería transmitir a los hombres y mujeres de todas las épocas. Como dichas desviaciones pueden producirse, entre otros ámbitos, a través de una terminología poco afortunada, resulta de especial interés analizar cómo se produce la traducción de determinados campos léxicos, desde el texto original al texto final.

Uno de estos campos lo constituye el correspondiente a la "mujer", que es el que nos va a ocupar a lo largo del presente trabajo. Desde

Futhark 8 (2013)

Ayerbe, Diferencias en la traducción, 9-30

ISSN 1886-9300 
luego, hay que comenzar poniendo de manifiesto que no faltan referentes femeninos de muy diversa tipología en el texto bíblico. Efectivamente, una lectura de los diferentes libros que componen el Antiguo y el Nuevo Testamento da cuenta de innumerables personajes femeninos, con nombre propio la mayoría, entre los que encontramos jóvenes y adultas; casadas, solteras, vírgenes y viudas; madres, hijas, cuñadas, suegras y nueras; mujeres de buena y mala reputación; santas y pecadoras; discretas y heroínas, etc.

Como es obvio, en cada lengua se cuenta con un inventario de unidades léxicas o términos, que pertenecen a un mismo campo léxico, y que son utilizados para aplicarlos en función del tipo de referente en cada caso. Así, se puede hacer alusión a una mujer soltera o casada, a una joven o a una mujer madura, todo ello en función del término que se escoja. Del mismo modo, se puede hacer referencia a una misma mujer, según la perspectiva desde la que se menciona, utilizando para ello uno $\mathrm{u}$ otro término. En este último sentido, a una misma mujer se la puede denominar en diferentes contextos "esposa", "madre", "joven", "muchacha", "sierva", etc.

Con todo ello queda esperar, en consecuencia, que para un contexto determinado siempre se utilizará un mismo término, de manera que, en la medida de lo posible, haya la posibilidad de establecer una relación 1:1. Por poner un ejemplo, sería de esperar que para hacer alusión a toda mujer que ha dado a luz se utilice siempre el término "madre" en castellano, "mother" en inglés, "mor" en noruego o "Mutter" en alemán.

Ahora bien, en el análisis del campo léxico "mujer" en lenguas germánicas antiguas se constatan indicios de que lo anteriormente dicho no siempre es así. En otras palabras, en textos bíblicos -ante todo neotestamentarios- parece observarse distinciones en el uso de uno u otro término, no precisamente según el contexto de que se trate, como sería de esperar, sino según el referente, es decir, dependiendo de la mujer concreta de la que se está hablando en un momento determinado. Para ser más exactos, estudios previos en alto alemán antiguo (Ayerbe 2011a), en alemán medio (Ayerbe 2011b) y en sajón antiguo (Ayerbe 2012) parecen demostrar que al hacer alusión a mujeres en los diferentes textos analizados, se utiliza distinta terminología según sea el referente la Virgen María, por un lado, o cualquier otra mujer, por otro lado, incluso cuando el contexto es el mismo. Más concretamente, es el contexto relativo al significado "esposa, mujer casada" donde las distinciones a las que nos referimos resultan más productivas. Así, por ejemplo, hay términos que se utilizan exclusivamente cuando el referente es la Virgen María, mientras que otros se evitan para hacer alusión también a ella.

Ahora bien, si la Virgen María en esta tierra fue niña, joven, mujer, esposa, madre, virgen, viuda, como lo puede ser cualquier otra mujer,

Futhark 8 (2013)

Ayerbe, Diferencias en la traducción, 9-30

ISSN 1886-9300 
se plantea entonces el interrogante acerca de los motivos que pudieron llevar a los autores de traducciones y otros textos en lenguas germánicas antiguas a llevar a cabo dichas distinciones. En nuestra modesta opinión, la respuesta a este interrogante podría hallarse en la siguiente hipótesis que, una vez más, proponemos para su discusión: efectivamente, la Virgen María es un ser de sexo femenino, es madre, es esposa, es virgen, es viuda, fue niña, fue joven, fue mujer adulta. Todo ello, ciertamente, se puede predicar igualmente de cualquier otra mujer, mas hay un rasgo que la hace a ella singular: si bien todos los atributos que hemos enumerado ya un par de veces se pueden ciertamente predicar de cualquier otra mujer, sólo de la Virgen María, sin embargo, se pueden predicar simultáneamente "mujer casada", "virgen" y "madre", sobre todo los dos últimos. Y ello, sólo en virtud de un particularísimo y exclusivo privilegio divino, según el cual la Virgen María, y únicamente ella, sería madre sin dejar por ello de ser virgen antes, durante y después del parto. Como se puede deducir, dicho privilegio singularizaba a la Virgen María respecto de las demás mujeres que habitaron y habitarán la tierra, y constituía al mismo tiempo la materia de una enseñanza teológica de capital trascendencia para los cristianos, que había que dejar a salvo de cualquier ambigüedad o errónea interpretación.

Como es lógico, este propósito debía hallar eco, entre otros, también en el campo de la transmisión literaria escrita, mediante la distinción lingüística en la elección de expresiones terminológicas adecuadas. En este sentido, para referirse, por ejemplo, a una legítima "esposa", como el común de los mortales espera que una esposa normal y corriente tenga relaciones conyugales con su marido, así como que conciba y dé a luz a raíz de éstas, la utilización de la misma terminología tanto para una mujer corriente como también para la Virgen María podría difuminar su perpetua virginidad, a pesar de su maternidad, o llevar a confusión. Téngase en cuenta que las enseñanzas y dogmas de la doctrina cristiana no estaban entonces tan desarrollados ni contaban con la misma difusión y fundamentación que en nuestros días ${ }^{2}$.

2 Efectivamente, aunque puntos fundamentales de la doctrina ortodoxa sobre la Virgen María se fueron definiendo ya en los primeros siglos de nuestra era, las discusiones sobre éstos se extendieron, sin embargo, durante los siguientes siglos hasta que quedaron definitivamente establecidos y asentados por las sucesivas autoridades apostólicas. Así por ejemplo, la Maternidad divina de María ya fue profesada en el siglo IV (cfr. Bastero 1995: 200 y s.), siglo en el que también tuvo lugar la traducción de la Biblia al gótico por Úlfilas. Asimismo, la doctrina sobre la perpetua virginidad de María fue también defendida y definida a lo largo del siglo IV y V (cfr. Bastero 1995: 220 y s.), donde jugaron un papel relevante la Epístola Dogmática del papa san León Magno Lectis dilectionis tuae a Flaviano (13.VI.449) y el Concilio Ecuménico de Calcedonia (8.X.451-1.XI.451), ambos acontecimientos un siglo después de la existencia y labor de Úlfilas.

Futhark 8 (2013)

Ayerbe, Diferencias en la traducción, 9-30

ISSN 1886-9300 
Así pues, a través de una apropiada distinción terminológica, sería posible hace entender de algún modo el lector la singularidad de la Virgen María respecto de las demás mujeres. En otras palabras, al emplear un término determinado para referirse a la Virgen María, por ejemplo, como esposa, se está dando a entender con ello que ella tiene alguna cualidad especial que la debe distinguir de otras mujeres también, como esposas. En este sentido, pensamos que dichas distinciones terminológicas no fueron fortuitas, sino que más bien eran intencionadas por parte de los autores y traductores de los diferentes textos.

\section{Objetivos y procedimiento}

En el presente trabajo intentaremos ofrecer alguna respuesta a los siguientes interrogantes: en primer lugar, ¿se aprecia un uso diferente de términos léxicos en griego, según sea el referente la Virgen María u otra mujer?, ¿sucede algo parecido en la lengua gótica?, ¿se utiliza siempre el mismo término en gótico para traducir la misma expresión del griego?

Para este fin, se llevó a cabo, en primer lugar, un rastreo terminológico de todos los términos pertenecientes al campo léxico "mujer" a lo largo de los fragmentos conservados de la Biblia traducida al gótico por el obispo Ulfila. En esta fase resultó especialmente útil contar con la ayuda del rastreador léxico facilitado por el banco de textos del Thesaurus Indogermanischer Text- und Sprachmaterialien (T.I.T.U.S.). El rastreo fue realizado teniendo en cuenta la flexión gramatical de cada uno de los términos buscados, por lo que hubo que introducir en el rastreador el mismo término con sus correspondientes sufijos de número y caso.

Una vez obtenidos los resultados de las diferentes búsquedas léxicas, se procedió a emparejar cuidadosamente cada término hallado en gótico con su correspondiente en el texto original, es decir, en griego. La finalidad de este modo de proceder era comprobar hasta qué punto se podía establecer una relación estricta 1:1 entre términos griegos y sus correspondientes traducciones en el texto gótico.

En una fase posterior, se volvió a repasar el corpus recogido de términos del campo léxico "mujer", atendiendo a sus referentes, esto es, a la(s) mujer(es) concreta(s) para la(s) que se empleaba cada término. Este procedimiento nos permitiría descubrir si se daba algún tipo de discriminación o restricción en cuanto al uso de uno u otro término, según la mujer a la que se estuviera haciendo referencia en cada momento. Más concretamente, se trataba de comprobar hasta qué punto había

3 El banco de textos es accesible en la dirección http://titus.unifrankfurt.de/indexs.htm?/index2.htm\#Dstart (última consulta: 02.02.2012).

Futhark 8 (2013)

Ayerbe, Diferencias en la traducción, 9-30

ISSN 1886-9300 
algún tipo de restricción en el uso de los diferentes términos léxicos del campo léxico "mujer", según fuera la Virgen María, por un lado, u otra mujer, por otro, el referente. El procedimiento se aplicó tanto a los términos griegos como a los góticos.

El corpus de textos sobre el que se ha llevado a cabo el presente estudio se reduce a los fragmentos conservados de la traducción de la Biblia del griego al gótico, obra del obispo arriano Úlfilas. Por desgracia, no contamos con más textos escritos en esta misma lengua en la misma época, salvo el Skeireins, un comentario de composición original al Evangelio de san Juan, el cual, para ser exactos, sólo aportó una única muestra del término qino, empleado para referirse a mujeres en gene$\mathrm{ral}^{4}$.

\section{Relación de muestras léxicas e índices de ocurrencias}

A continuación se presenta el inventario de términos góticos para el campo léxico "mujer", hallados en el texto traducido de la Biblia. Los términos góticos aparecen en la columna de la izquierda; en la columna siguiente se añade el término del texto griego original que se traduce; a continuación, en otra columna se indica el significado en castellano y, finalmente, se indica en la columna de la derecha el número total de ocurrencias en el texto:

\begin{tabular}{|c|c|c|c|}
\hline Término gótico & $\begin{array}{l}\text { Término } \\
\text { griego del origi- } \\
\text { nal }\end{array}$ & Significado & Ocurrencias \\
\hline barn & таĨ & "niña" & 4 \\
\hline brups $^{5}$ & vú $\mu \varphi \eta$ & "nuera" & 3 \\
\hline magaps & пар日⿱一⿻上丨 & "virgen" & 2 \\
\hline mawi & 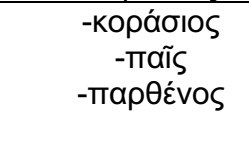 & $\begin{array}{l}\text {-"muchacha", } \\
\text { "niña" } \\
\text {-“niña" } \\
\text {-"virgen" }\end{array}$ & $\begin{array}{l}6 \\
2 \\
3\end{array}$ \\
\hline
\end{tabular}

$4 \quad$ El pasaje en cuestión es el siguiente: “(...) fimf pusundjos waire inuh qinons jah barna (...)" (VII, 8-9). "(...) cinco mil hombres, sin contar mujeres ni niños (...)". La localización de la cita se basa en la edición de Streitberg, del año 2000. Esta cita concreta se refiere al episodio de la multiplicación milagrosa de los panes y los peces, recogida en $\mathrm{Jn} \mathrm{VI}, 1-15$, aquí concretamente, $\mathrm{VI}, 10$.

5 Aunque es probable que brups, según Braune (1907) tuviera más significados en gótico, además del de nuera, nos hemos limitado aquí a consignar únicamente el que hemos podido constatar en el texto consultado. En cualquier caso, significando "nuera", es evidente que la mujer a la que se hace referencia con él debe ser una mujer casada.

Futhark 8 (2013)

Ayerbe, Diferencias en la traducción, 9-30

ISSN 1886-9300 


\begin{tabular}{|c|c|c|c|}
\hline qens & yuvń & $\begin{array}{l}\text { "mujer casa- } \\
\text { da" }\end{array}$ & 40 \\
\hline qino & yuvń & $\begin{array}{l}\text { "mujer (gené- } \\
\text { rico)" "ser hu- } \\
\text { mano adulto de } \\
\text { sexo femenino" }\end{array}$ & 38 \\
\hline piwi & 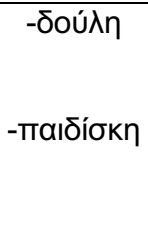 & $\begin{array}{l}\text {-"sierva, es- } \\
\text { clava, persona } \\
\text { sometida a otra" } \\
\text {-"muchacha", } \\
\text { "esclava", "sir- } \\
\text { vienta (en una } \\
\text { corte)" }\end{array}$ & 10 \\
\hline
\end{tabular}

Tabla 1: Muestras del campo léxico "mujer" en gótico, con los términos originales en griego y el número de muestras recogidas.

En la tabla 1 se recogen las muestras en gótico en caso nominativo y número singular. Ahora presentamos nuevamente dichas muestras en todas las variantes flexivas constatadas para referirse exclusivamente a "seres humanos de sexo femenino"6.

-barn, barnis

$-b r u p$

-magapai, magapais

-mauja, maujai, maujos, mawi, mawilo

-qenai, qenais, qeins, qenins, qens

-qino, qinom, qinon, qinono, qinons

-piujai, piujo, piujos, piwi

$6 \quad$ Esta aclaración, que podría parecer a primera vista un tanto superflua, nos vemos obligados a hacerla, ya que al menos el término barn podía utilizarse en gótico para referirse tanto a una niña como a un niño, tal como se puede comprobar en las siguientes citas, referidas concretamente a Juan el Bautista: jah warb, swe hausida Aileisabaib golein Mariins, lailaik barn in qibau izos, [...] $(\mathrm{Lc} I, 41)$ "En cuanto Isabel oyó el saludo de María, se agitó el niño en su vientre"; sai allis, sunsei warb stibna goleinais peinaizos in ausam meinaim, lailaik pata barn in swignipai in wambai meinai. (Lc I,44) "Justo en el instante en el que la voz de tu saludo llegó hasta mis oídos, saltó de gozo el niño en mi seno".

Futhark 8 (2013)

Ayerbe, Diferencias en la traducción, 9-30

ISSN 1886-9300 


\section{Análisis individual del uso de cada término en gótico}

\subsection{Barn}

Este término se utilizaba para hacer alusión a un menor de corta edad, es decir, a alguien en edad aún infantil hasta las puertas de la adolescencia. Se trata de un substantivo común que se puede emplear tanto para alguien de sexo masculino como femenino. El sustantivo se utiliza para traducir el griego maĩs "niño/a". Referido a personas de sexo femenino, tan sólo hemos hallado cuatro muestras, referidas todas ellas a la hija de Jairo, el jefe de la sinagoga, en el Evangelio de san Marcos:

1) (...) jah innatgaggands qap du im: ha auhjop jah gretip? pata barn ni gadaupnoda, ak slepip. jah bihlohun ina. ip is uswairpands allaim ganimip attan pis barnis jah aipein jah pans mip sis. jah galaip inn parei was pata barn ligando. jah fairgraip bi handau pata barn qapuh du izai: $(\ldots)^{7}$ (Mc V, 39-41) “(...) y entrando, les dijo: "¿A qué vienen estos gritos y llantos? La niña no está muerta, sino dormida. Mas ellos se rieron de él. Pero él los mandó fuera a todos, $y$ tomando al padre y a la madre de la niña, y a los que venían con él, entró en la habitación en la que se encontraba la niña. Y tomando a la niña de la mano, le habló diciendo: (...)"8

La edad de la niña en cuestión no es difícil de averiguar, pues en el versículo 42 del mismo Evangelio se indica que tenía doce años "(...) was auk jere twalibe;". Fuera de este episodio, no hemos encontrado más muestras referidas a una mujer, aunque ello no nos impide conjeturar que no habría habido objeción alguna a su uso para la Virgen María, si hubiera aparecido en el texto neotestamentario descrita como una niña de corta edad, pues obviamente también ella tuvo que haber sido niña en la primera etapa de su vida.

\subsection{Brups}

La única muestra encontrada de este término se halla en el Evangelio de san Mateo:

2) qam auk skaidan mannan wipra attan is jah dauhtar wipra aipein izos jah brup wipra swaihron izos; $(. .).(\mathrm{X}, 35)$ "He venido a dividir al hombre contra su padre, y a la hija contra su madre, y a la nuera contra su suegra; (...)"

7 En las citas he respetado los signos de puntuación así como el uso de mayúsculas y minúsculas de la edición del texto original de Streitberg (2000).

8 Todas las traducciones al castellano de las citas en lengua gótica son obra del autor del presente trabajo.

Futhark 8 (2013)

Ayerbe, Diferencias en la traducción, 9-30

ISSN 1886-9300 
De lo que no queda duda, debido al contexto, es que brups, en cuanto a su uso, hace referencia explícita a una mujer casada, y traduce el término griego vú $\mu \varphi \eta$ "nuera".

\subsection{Magaps}

La palabra designa a una mujer virgen en el sentido estricto de la expresión. En la Biblia gótica sólo aparece para referirse a la Virgen María,

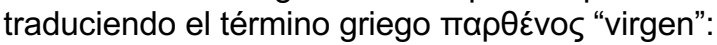

3) panuh pan in menop saihstin insandips wa aggilus Gabriel fram guda in baurg Galeilaias sei haitada Nazaraip. du magapai in fragibtim abin, pizei namo losef, us garda Daweidis, jah namo pizos magapais Mariam. (LC I, 2627) "En el sexto mes fue enviado el ángel Gabriel a una ciudad de Galilea, llamada Nazaret, a una virgen desposada con un varón, de nombre José, de la casa de David. Y el nombre de la virgen era María."

Fuera de ella, el término magaps no se constata empleado para referirse a otra mujer.

\subsection{Mawi}

Este término se utiliza en la traducción gótica para referirse a una niña o muchacha joven. Dicha niña puede encontrarse a las puertas de la adolescencia, saliendo ya de la edad infantil, o bien en plena adolescencia o al final de ella. En el texto de la Biblia gótica lo encontramos para referirse a dos personas: en primer lugar, a la hija de Jairo, el jefe de una sinagoga judía, una niña de doce años a la que Jesús resucita y devuelve a sus padres:

4) afleipip, unte ni gaswalt so mawi, akslepip. jah bihlohun ina. panuh pan usdribana warp so managei, atgaggands inn habaida handu izos, jah urrais so mawi. (LC IX, 24-25) "¡Salid fuera, pues la niña no está muerta, sino durmiendo! Y se rieron de él. Después de haber echado a la gente, entró y la tomó [a la niña] de la mano. Y la niña se levantó."

5) qimands pan in garda ni fralailot ainohun inngaggan, alja Paitrau jah lakobu jah lohannen jah pana attan pizos maujos jah aipein. (LC VIII, 51) "Entrando en la casa no dejó pasar a nadie sino sólo a Pedro, Santiago y Juan, así como al padre de la muchacha y a la madre."

El término traduce aquí dos expresiones distintas del texto original griego, concretamente maĩs y корáбıos; en segundo lugar, el término se emplea también para hacer alusión a la hija de Herodías, la joven que sale a bailar para divertir a los invitados al banquete de Herodes:

6) jah atgaggandein inn dauhtar Herodiadins jah plinsjandein jah galeikandein Heroda jah paim mipanakumbjandam, qap piudans du pizai maujai: bidei mik

Futhark 8 (2013)

Ayerbe, Diferencias en la traducción, 9-30

ISSN 1886-9300 
pishizuh pei wileis, jah giba pus. (Mc VI, 22) "Entró entonces la hija de Herodías y se puso a bailar. Y gustó tanto a Herodes y a sus invitados, que dijo a la muchacha: 'Pídeme lo que quieras y te lo daré."'

7) jah atbar pata haubip is ana mesa jah atgaf ita pizai maujai, jah so mawi atgaf ita aipein seinai. (Mc VI, 28) "Y trajo su cabeza [de Juan el Bautista] sobre una bandeja y se la entregó a la muchacha; y la muchacha se la entregó a su madre."

En el caso de la hija de Herodías, ésta aparece en el texto griego únicamente como kopáoı ésta sería algo mayor que la de la hija de Jairo.

Ahora bien, mawi no sólo se emplea para referirse a una niña o a una muchacha más adulta. Su uso se constata también para hacer alusión a una virgen. A este respecto, llama la atención que las muestras halladas en la Biblia gótica únicamente se refieran a mujeres que son vírgenes, salvo para la Virgen María:

8) appan jabai nimis qen, ni frawaurhtes, jah jabai liugada mawi, ni frawaurhta, (...) (I Cor VII, 28) "Mas si tomas esposa, no pecas; y si una virgen se casa, tampoco peca."

9) appan bi maujos anabusn fraujins ni haba; (...) (I Cor VII, 25) "En cuanto a las vírgenes, no tengo precepto alguno de parte del Señor; (...)"

10) (...) gawadjoda auk izwis ainama waira mauja swikna du usgiban Xristau. (II Cor XI, 2) " (...) y os prometí a un único hombre para entregaros como una virgen pura a Cristo."

En las tres últimas citas, 8,9 y 10, mawi se emplea para traducir el tér-

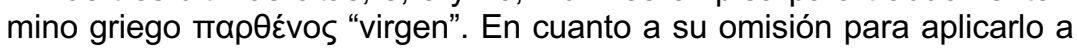
la Madre de Dios, que también es virgen, trataremos esta cuestión más adelante, al objeto de no salirnos del propósito de esta sección, consistente en presentar las muestras halladas en gótico para traducir términos del campo léxico "mujer" del griego.

\subsection{Qens}

Junto con qino, este término es el que cuenta con el mayor índice de ocurrencias en el texto gótico. Por las mujeres a las que hace referencia expresa, así como por los contextos en los que aparece, se puede concluir sin lugar a dudas que la expresión qens se utiliza para referirse a una mujer legítima y verdaderamente casada. Traduce el término griego yuvń. Ejemplos especialmente ilustrativos podemos encontrar en la exposición del caso de los siete hermanos que, casándose sucesivamente con la viuda, fallecen sin dejar descendencia:

Futhark 8 (2013)

Ayerbe, Diferencias en la traducción, 9-30

ISSN 1886-9300 
11) du atgaggandans pan sumai Saddukaie, paiei qiband usstass ni wisan, frehun ina qipandans: laisari, Moses gamelida uns, patei jabai his bropar gadaupnai aigands qen, jah sa unbarnahs gadaupnai, ei nimai bropar is po qen jah urraisjai fraiw bropr seinamma. sibun nu broprjus wesun, jah sa frumista nimands qengadaupnoda unbarnahs. jah nam anpar po qen, jah sa gaswalt unbarnahs. jah pridja nam po samaleiko; samaleiko pan jah pai sibun, jah ni bilipun barne jah gaswultun. Spedista allaize gadaupnoda jah so qens. in pizai usstassai nu, harjis pize wairpip qens? pai auk sibun aihtedun po du qenai. (LC XX, 27-33) "Se presentaron entonces unos saduceos -que niegan la resurrección- y le preguntaron diciendo: 'Maestro, Moisés nos dejó mandado que si un hombre, que tiene un hermano, muere dejando una mujer sin hijos, su hermano tomará a la mujer y dará descendencia a su hermano. Pues bien, hubo una vez siete hermanos, y tomando el primero esposa, murió sin dejar hijos. Y el segundo tomó a la mujer y también éste murió sin dejar hijos. Del mismo modo, la tomó el tercero; y así los siete, muriendo sin dejar hijos. Después de todos ellos, murió también la mujer. En la resurrección, ¿de quién será entonces mujer? Pues los siete la tuvieron por esposa."

Hay, no obstante, otros muchos casos no menos ilustrativos, en los que se hace una referencia explícita a mujeres casadas:

12) was in dagam Herodes piudanis ludaias gudja namin Zakarias, us afar<am> Abijins, jah qeins i sus dauhtrum Aharons, jah namo izos Aileisabaip. (LC I, 5), donde se hace referencia a Isabel, la esposa de Zacarías.

13) sitandin pan imma ana stauastola, insandida du imma qens is qipandei: $\mathrm{ni}$ waiht pus jah pamma garaihtin ... (...) (Mt XXVII, 19) aludiendo a la esposa de Pilato.

14) (...) jah lohanna, qens Kusins, fauragaggjins Herodes, (...) (Lc VIII, 3) en alusión a Juana, la mujer de Cusa, el administrador de Herodes.

15) gamuneip qenais Lodis. (Lc XVII, 32), acerca de la esposa de Lot.

16) sa auk raihtis Herodes insandjands gahabaida lohannen jah gaband ina in karkari in Hairodiadins qenais Filippaus broprs seinis, unte po galiugaida. qap auk lohannes du Heroda patei ni skuld ist pus haban qen broprs peinis. (Mc VI, 17-18) "Herodes había mandado detener a Juan [el Bautista] y lo hizo encerrar en prisión a causa de Herodías, la esposa de su hermano Filipo, con la que se había casado. Juan le decía: 'No te es lícito tener a la esposa de tu hermano."

Claramente, el uso de qens está limitado a la consideración de una mujer como legítimamente casada con un hombre, con los consiguientes derechos y obligaciones. Esto había de entenderse tanto en un sentido civil y social como ante la ley divina.

Con todo, el uso de qens está también atestiguado para la Virgen María, como legítima esposa de san José: 
17) Urran ban jah losef us Galeilaia, us baurg Nazaraip, in ludaian, in baurg Daweidis sei haitada Bepla<i>haim, dupe ei was us garda fadreinais Daweidis, anameljan mip Mariin sei in fragiftim wa imma qeins, wisandein inkilpon. (Lc II, 4-5) "Y así pues, salió también José de Galilea, de la ciudad de Nazaret, hacia Judea, a la ciudad de David, llamada Belén, pues era él de la estirpe de David, para inscribirse junto con María, su esposa, que se hallaba encinta."

Ahora bien, la consideración de la Virgen María como esposa la trataremos en detalle más tarde.

\subsection{Qino}

El término hace referencia a una mujer de forma genérica y se utiliza para traducir la expresión griega yuvń, exclusivamente. El término qino, como decimos, puede referirse a cualquier mujer, que puede ser soltera, casada o viuda. Puede referirse también a una madre, a una enferma o, incluso, a una pecadora pública (mujer de mal vivir o prostituta). En cuanto a la edad, después de analizar todas las muestras recogidas en el texto, qino parece referirse exclusivamente a mujeres adultas, pues no hemos hallado muestras que hicieran alusión a mujeres de corta edad. La expresión se constata tanto para hacer alusión a la Virgen María, como a cualquier otra mujer. A continuación, citamos algunos ejemplos:

Para referise a la Virgen María:

18) (...) piupido pu in qinom, jah piupido akran qipaus peinis. (LC I, 42) "Bendita tú entre todas las mujeres y bendito el fruto de tu vientre". En este caso, Isabel se dirige con estas palabras a su prima, la Virgen María, por haber creído lo que le había dicho el arcángel Gabriel en el episodio de la Anunciación.

19) ip bipe qam usfulleins melis, insandida gup sunu seinana, waurpanana us qinon, waurpanana ut witoda, (...) (Gal IV , 4) "Y al cumplirse el tiempo, envió Dios a su hijo, nacido de una mujer y sometido a la Ley, (...)". Aquí la alusión a la Virgen María es indirecta.

Para referirse a una mujer cualquiera, en sentido general:

20) jah qino wisandei in runa blopis jera twalif, soei in lekjans fraqam allamma aigina seinamma, jah ni mahta was fram ainomehun galeikinon, (...) (Lc VIII, 43) "Entre la gente había una mujer que sufría de hemorragias desde hacía doce años, sin que nadie pudiera curarla, (...)"

21) aippau suma qino drakmans habandei taihun, jabai fraliusip drakmin ainamma, niu tandeip lukarn jah usbaugeip razn jah sokeip glaggwaba, unte bigitip? (Lc XV, 8) "O, si una mujer tiene diez dracmas y pierde una de ellas, 
¿acaso no enciende una lámpara, barre la casa y busca minuciosamente hasta que la encuentra?"

22) unte jabai ni huljai sik qino, skabaidau; ip jabai agl ist qinon du kapillon aippau skaban, gahuljai ... (...) (I Cor XI, 6) "Si una mujer no se cubre [la cabeza], que se corte el pelo; ahora bien, si para la mujer es vergonzoso cortarse el pelo, que se cubra [la cabeza]... (...)"

23) appan ik qipa izwis, patei hazuh saei saihuip qinon du luston izos, ju gahorinoda izai in hairtin seinamma. (Mt $\mathrm{V}, 28$ ) "Pues yo os digo que quien mira a mujer desándola, ya cometió adulterio con ella en su corazón."

\section{Para referirse a una mujer casada:}

24) jah jabai qino afletip aban seinana jah liugada anparamma, horinop. (Mc $X, 12)$ " $Y$ si una mujer abandona a su marido y se casa con otro, comete adulterio."

25) jus qinons, ufhausjaip wairam izwaraim, swe gaqimip in fraujin. (Col III,

18) "Esposas, sed sumisas a vuestros maridos, que así place al Señor."

Para hacer alusión a una viuda:

26) (...) jah ni du ainaihun pizo insandips was Helias, alja in Saraipta Seidonais du qinon widuwon. (Lc IV, 26) "(...) mas a ninguna de ellas fue enviado Elías, salvo a una mujer viuda de Sarepta, en Sidón."

Para referirse a una madre:

27) qino, pan bairip, saurga habaid, unte qam heila izos; (...) (Jn XVI, 21) "Cuando una mujer está a punto de dar a luz, pasa miedo porque ha llegado su hora; (...)"

28) gahausiandei raihtis qino bi ina, pizozei habaida dauhtar ahman unhrainjana, qimandei draus du fotum is. (Mc VII, 25) "Oyendo hablar de él una mujer que tenía una hija poseída por un espíritu inmundo, vino enseguida y se postró a sus pies."

Para referirse a una pecadora/adúltera:

29) paruh sai, qino in pizai baurg, sei was frawaurhta, jah ufkunnandei patei anakumbida in razna pis Fareisaiaus, (...) (Lc VII, 37) "Y al enterarse una mujer de aquella ciudad, que era pecadora, que [Jesucristo] había ido a la casa del fariseo, (...)"

30) gasaihuands pan sa Fareisaius, saei haihait ina, rodida sis ains qipands: sa ip weisi praufetus, ufkunpedi pau, ho jah hileika so qino sei tekip imma, patei frawaurhta ist. (Lc VII, 39) "Viendo esto el fariseo que le había invitado, pensó para sí: 'si éste fuera un profeta se daría cuenta de la clase de mujer que le está tocando; sabría que es una pecadora."”

Futhark 8 (2013)

Ayerbe, Diferencias en la traducción, 9-30

ISSN 1886-9300 


\subsection{Diwi}

El uso de esta palabra está limitado a toda mujer, con independencia de su edad, que desempeña la función de sirvienta o criada. También lo usa una mujer que se considera a sí misma, en su humildad, sierva o esclava de alguien. El término piwi se emplea en gótico para traducir

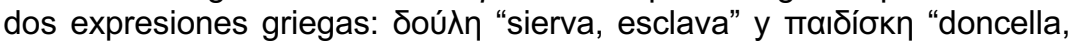
esclava, cortesana". De todas las muestras recogidas, sólo en dos ocasiones hace referencia piwi a la Virgen María, mientras que en el resto de los casos hace alusión a otras mujeres. Tanto la Virgen María como las demás mujeres a las que se aplica este término tienen en común el ser consideradas -o considerarse a sí mismas- esclavas o siervas. Ahora bien, no obstante lo dicho, la clasificación de las muestras recogidas permitía constatar que piwi traducía el término griego ठoú $\lambda \eta$ cuando éste se refería a la Virgen María, así como maıסíokn cuando éste se refería a otra mujer. Veamos algunos ejemplos:

Para referirse a la Virgen María:

31) qap ban Mariam: sai, piwi fraujins, wairbai mis bi waurda peinamma. (Lc I, 38) "Y dijo entonces María: 'He aquí la esclava del Señor, hágase en mí según tu palabra". María, ante el anuncio de la extraordinaria misión que recibe de parte de Dios, se considera humildemente su esclava.

32) unte insah du hnaiweinai piujos seinazos; (...) (Lc I, 48) "Y miró la humildad de su esclava". Todo ello palabras de la misma Virgen María, expresando cómo se sentía ante la majestad y la inmensidad de Dios, que se fija en ella.

Para referirse a otras mujeres, también en la función de siervas:

33) jah wisandin Paitrau in rohsnai dalapa [jah $]^{9}$ atiddja aina piujo pis auhumistins gudjins, (...) (Mc XIV, 66) "Estando Pedro abajo, en el patio, se acercó una de las criadas del Sumo Sacerdote, (...)"

34) ip Paitrus uta sat ana rohsnai, jah duatiddja imma aina piwi qipandei: (...) (Mt XXVI, 69) "Pedro estaba sentado en el patio y se le acercó una criada diciendo: $(\ldots)$

35) paruh qap jaina piwi, so daurawardo, du Paitrau: (...) (Jn XVIII, 17) "Y entonces dijo una criada -la portera- a Pedro: (...)"

36) gamelip ist auk patei Abraham twans sununs, ainana us piujai jah ainana us frijai; akei pan sa us piujaik bi leika ... (...) (Gal IV, 22-23) "Está dicho que Abraham tuvo dos hijos, uno de la esclava y uno de la libre. El de la esclava, de forma natural ... (...)"

$9 \quad$ El añadido entre paréntesis es de la edición del texto original. Futhark 8 (2013) Ayerbe, Diferencias en la traducción, 9-30 ISSN 1886-9300 


\section{Interpretación de resultados}

El análisis de las muestras recogidas, así como de la función que desempeñan en el texto gótico, permiten constatar algunos hechos, lo cuales exponemos a continuación.

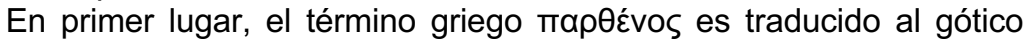
con dos términos distintos: magaps cuando se habla de la Virgen María y mawi cuando la virgen en cuestión es otra mujer. Ambos términos góticos muestran, por tanto, una distribución complementaria, de suerte que magaps se constata únicamente para referirse a la María como virgen, mientras que el otro sólo se constata cuando la virgen a la que se hace alusión es otra mujer. Esta diferenciación terminológica no se

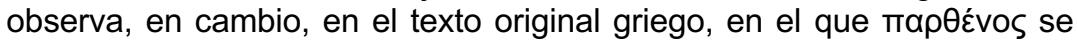
emplea siempre para hacer referencia a una virgen, ya se trate de María como de otra mujer de la misma condición.

Llegados a este punto, cabe plantearse el motivo por el que se utilizan en gótico dos términos distintos para referirse a un mismo estado, concretamente, el de la virginidad. Más llamativa, si cabe, hace esta distinción el constatar que ambos términos góticos traducen un único

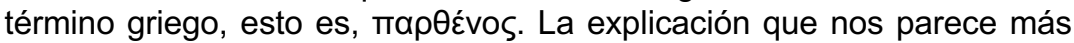
plausible aquí se basa en la consideración de que magaps era más restrictivo en cuanto a su significado y, por tanto, también en lo que afecta a su referente. Efectivamente, magaps es un término limitado a una virgen o célibe, mientras que mawi no tenía una matriz semántica tan restrictiva. Así pues, mawi podía significar "virgen", pero no de manera exclusiva, pues también podía hacer referencia a una doncella, una muchacha, una niña, una joven, etc, que, efectivamente, podía incluir además la condición de virgen, pero no de forma obligatoria, sino como una más. De hecho, mientras magaps se emplea únicamente para tra-

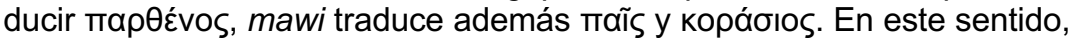
y dada la multiplicidad de contextos y funciones en la que se puede encontrar mawi, el rasgo semántico de la virginidad podría pasar, pensamos, algo más inadvertido o diluido, algo que no ocurre con magaps, término en el que la virginidad es el rasgo semántico esencial. Así pues, el empleo de magaps para hacer alusión exclusivamente a la Virgen María no haría sino resaltar una característica personal de enorme importancia: la de su perpetua virginidad, sin dejar lugar a dudas ni ambigüedades. Hablar de María como doncella, joven o muchacha (mawi) podría, desde luego, haber dejado lugar a una posible pero remota interpretación de su persona como virgen, pero no de un modo tan claro y definitivo como magaps.

Algo similar, pero en relación inversa, se observa en los términos para referirse a una sierva o criada. Para este fin se constatan dos térmi-

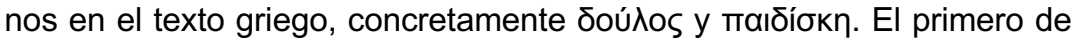

Futhark 8 (2013)

Ayerbe, Diferencias en la traducción, 9-30

ISSN 1886-9300 
ellos aparece empleado por la misma Virgen María para hablar de sí misma como "sierva/esclava del Señor", mientras que el segundo se utiliza para referirse a cualquier otra sierva. Ahora bien, la cuestión es que en el texto meta, el gótico, ambos términos griegos son traducidos por el mismo término gótico, esto es, piwi. En este caso, nos encontramos con que el texto gótico no establece distinción terminológica alguna en función del referente.

En cuanto al término qens, éste se emplea sin lugar a dudas para referirse a una mujer legítimamente casada, como decíamos antes. El uso del término ha sido constatado para hacer alusión tanto a la Virgen María como a otras mujeres, en cuanto mujeres casadas. Ahora bien, no obstante lo dicho, se observa una cierta matización cuando qens se utiliza para hacer referencia a la Virgen María como esposa, las cuales brillan por su ausencia, o al menos no parecen tan necesarias, cuando el referente es otra mujer, también casada legítimamente. En concreto, cuando el referente es la Virgen María, el sustantivo qens aparece acompañado de fragifts, que significa "desposada", y traduce el partici-

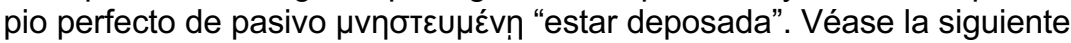
cita:

37) Urrann ban jah losef us Galeilaia, us baurg Nazaraip, in ludaian, in baurg Daweidis sei haitada Bepla<i>haim, dupe ei was us garda fadreinais Daweidis, anameljan mip Mariin sei in fragiftim was imma qeins, wisandein inkilpon. (LC II, 4-5) "Y así pues, salió también José de Galilea, de la ciudad de Nazaret, hacia Judea, a la ciudad de David, llamada Belén, pues era él de la estirpe de David, para inscribirse junto con María, su esposa, que se hallaba encinta."

La pregunta aquí sería por qué esta distinción entre María y otras mujeres, si tanto unas como otras tenían en común estar casadas. La explicación que podemos ofrecer aquí se basa en la hipótesis de que habría la intencionalidad de salvaguardar lingüísticamente que María, a pesar de ser una mujer verdaderamente casada, en su matrimonio no había cohabitación carnal como es de esperar en todo matrimonio legal y verdadero canónicamente. María y José eran verdaderos esposos ante Dios y los hombres pero, sin aplicar matiz alguno en el caso de María en cuanto a su matrimonio, quizá no hubiera quedado suficientemente resaltada su perpetua virginidad, dejándola a oscuras y, por tanto, abrien-

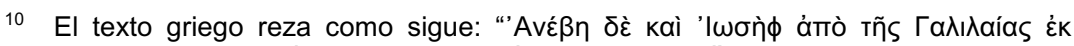

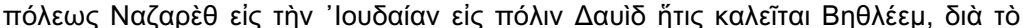

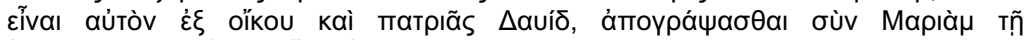

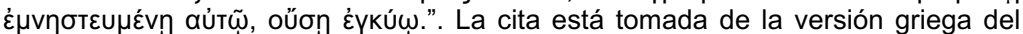
Nuevo Testamento, editada electrónicamente en el Thesaurus Indogermanischer Text- und Sprachmaterialien (T.I.T.U.S.), última consulta: 21.02.2012 <http://titus.uni-frankfurt.de/texte/etcs/grie/gnt/gnt.htm>.

Futhark 8 (2013)

Ayerbe, Diferencias en la traducción, 9-30

ISSN 1886-9300 
do puertas a confusiones en un aspecto doctrinal crucial y decisivo como era éste. Hay que tener en cuenta que en María se da, por un privilegio divino exclusivo, la circunstancia de ser madre y virgen simultáneamente, algo que en absoluto podía predicarse de otra mujer, por muy ejemplar y santa que fuera, y precisamente esta singularidad tenía que encontrar su reflejo también a nivel lingüístico. Como, muy probablemente, qens por sí solo no sería suficiente para ello, se añade fragi$\mathrm{fts}$, del mismo modo que en griego se añade el participio $\varepsilon \mu \vee \eta \sigma \tau \varepsilon \cup \mu \varepsilon \vee \eta$ a yuvń. He aquí, por tanto, una diferenciación más en cuanto al uso de términos, esta vez tanto en el texto griego original como en el texto gótico final, pues ambos utilizan el mismo término para referirse a una mujer casada, sea quien sea, con la salvedad de incluir el matiz léxico con valor semántico "desposada" únicamente en los casos en los que el referente es la Virgen María.

Otro argumento que refuerza la explicación aquí ofrecida es la aparición del matiz "desposada" también en el capítulo anterior del Evangelio de san Lucas, en el que se describe que el arcángel Gabriel fue enviado a una virgen "desposada" con un hombre de la casa de David, esto es, José:

38) panuh pan in menop saihstin insandips was aggilus Gabriel fram guda in baurg Galeilaias sei haitada Nazaraip, du magapei in fragibtim abin, pizei namo losef, (...) (Lc I, 26-27) "En el sexto mes fue enviado el arcángel Gabriel de parte de Dios a una ciudad de Galilea, llamada Nazaret, a una virgen desposada con un hombre, cuyo nombre era José, (...)"

Esta vez, el adjetivo fragifts no acompaña a qens, sino a magaps. En el texto griego, elparticipio $\mu \varepsilon \mu \vee \eta \sigma т \varepsilon \cup \mu \varepsilon ́ v \eta v$ acompaña, por su parte, a

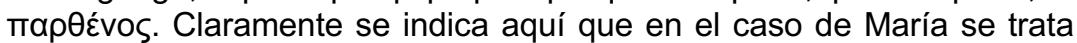
de una mujer legítimamente desposada, que al mismo tiempo es y permanece virgen, lo cual se busca resaltar de forma explícita desde un punto de vista lingüístico. De esta forma se consigue hacer simultáneo algo que en el resto de los casos es imposible predicar de otra mujer, salvo en casos muy aislados ${ }^{11}$ : ser esposa y permanecer virgen al mismo tiempo.

El término qino, por su parte, no presenta particularidades en cuanto a su uso. Efectivamente, su empleo no hace distinciones entre referentes, pudiéndose constatar tanto para hacer referencia la Virgen María, como a otras mujeres. La única salvedad es que el número de muestras

11 Ciertamente, sería perfectamente posible la situación en la que cualquier otra mujer se casara y al mismo tiempo decidiera, de común acuerdo con su legítimo esposo, permanecer virgen intacta. Si bien una situación así sería, como decimos, posible, se trataría de casos muy concretos y particulares, pues lo esperado entre un hombre y una mujer que desean contraer matrimonio es precisamente consumarlo en el acto conyugal y tener descendencia.

Futhark 8 (2013)

Ayerbe, Diferencias en la traducción, 9-30

ISSN 1886-9300 
referidas a la Virgen María es llamativamente menor que el de las muestras referidas a otras mujeres. Ahora bien, esta diferencia tiene su explicación: la Virgen María es una figura femenina más en los textos neotestamentarios, frente al elevado número de otras mujeres en las circunstancias más variadas. Y no es de extrañar que el término qino se emplee tanto para una como para otras, pues al ser un término genérico para referirse a un ser femenino adulto, se trata aquí de una matriz semántica que posee cualquier mujer, y tan mujer es la Virgen María como las demás que aparecen en el texto, tanto original como meta. No en vano, es el segundo término más veces constatado, a tan sólo dos muestras de diferencia por debajo de qens. Aparte de lo dicho, quedaría únicamente añadir que qino, además de referirse a una mujer en sentido genérico, dicha mujer es de edad adulta, pues no se constata su uso en menores, al menos en la traducción del texto bíblico.

En cuanto a los demás términos, barn y brups, el principal problema es la escasez de muestras recogidas como para estar en condiciones de proponer afirmaciones, pues cada uno de ellos fue constatado una única vez. Ahora bien, con lo visto hasta ahora, pensamos que nada impediría aventurar la hipótesis de que ambos serían compatibles tanto para la Virgen María como para otras mujeres. El primero de ellos, barn hace alusión a una niña, es decir, simplemente a un ser de sexo femenino y de muy corta edad. Sin duda, también la Virgen María había pasado biológicamente por la fase infantil, como todas las demás mujeres. Por otro lado, también brups podría haber sido empleado para hacer alusión a la Virgen María, pues siendo esposa legítima de san José, habría sido simultáneamente nuera, contando con que los padres de José aún siguieran con vida -algo de lo que, por cierto, los Evangelios no nos proporcionan constancia alguna- desde el momento en que María y José se convirtieran en esposos. A esto hay que añadir que ambos términos tampoco se constatan más veces para otras mujeres, lo cual nos permite concluir que ambos términos, barn y brups no aparecen más veces, no porque haya motivos de incompatibilidad tanto para la Virgen María como para otras mujeres, sino más bien porque no se presentan más situaciones en las que el uso de estos términos sea necesario.

Con todo, hay en el caso de brups afirmaciones de Braune (1907: $30,36-37)$ que dan motivo para pensar que brups sería incompatible para la Virgen María en gótico, pues este término no se referiría únicamente a la nuera sino también a la mujer legítimamente casada, y unida en cuerpo y alma a su marido. A este respecto, María estaba ciertamente legítimamente casada con José, pero ello excluía la cohabitación carnal. El problema aquí es, como decíamos más arriba, que sólo hemos constatado una única muestra en gótico, y que ésta se refiere sin lugar a dudas a una nuera. Ello no excluye, por supuesto, la posibilidad

Futhark 8 (2013)

Ayerbe, Diferencias en la traducción, 9-30

ISSN 1886-9300 
de que se utilizara también para referirse a una mujer recién casada o, incluso, ya con tiempo en el matrimonio, como indica Braune. Desde luego, lo que se observa al menos en alto alemán antiguo y en sajón antiguo, en cuanto al uso de términos similares (brût en alto alemán antiguo y brûd en sajón antiguo) da la razón a Braune, pero haría falta realizar más estudios en inglés antiguo y en nórdico antiguo.

\section{Conclusión}

A la vista de las muestras analizadas y de los resultados alcanzados, podemos concluir que existen claros indicios de una diferenciación en el uso de algunos términos del campo léxico 'mujer', en función de la mujer concreta que constituya el referente, es decir, si se trata de la Virgen María, por un lado, o de otra mujer, por otro. Esto lo hemos comprobado con claridad al observar que para la traducción de un mismo término

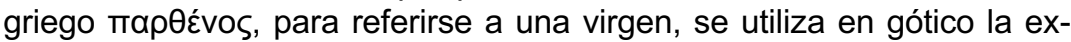
presión magaps exclusivamente para la Virgen María, mientras que para otra virgen se emplea mawi, excluyendo a la Virgen María. En cuanto a qens, para una mujer casada, hemos podido constatar que éste se utiliza tanto para la Virgen María como para otras mujeres legítimamente casadas. Ahora bien, hay que decir a este respecto que la única muestra recogida para la Virgen María incluye una matización expresiva para resaltar su condición de "desposada"12. Dicha matización se halla presente, curiosamente, tanto en el texto griego como en el gótico. Como decíamos antes, en nuestra modesta opinión, esta matización no tendría más objeto que dejar claro a nivel lingüístico que, por un lado, María era legítima esposa, pero que al mismo tiempo, por otro lado, sólo estaba casada pero no había cohabitación carnal con José.

En lo que se refiere al significado "doncella, sierva, esclava", hemos constatado que en el texto griego se hace una distinción, dependiendo

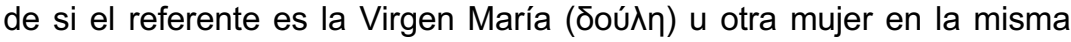

12 A este respecto hubiera sido interesante comprobar cómo habría sido traducido el pasaje recogido en Mateo I, 20, en el que el ángel se aparece en sueños a José para confirmarle que Dios cuenta con él para ser el legítimo esposo de María, a pesar de no haber intervenido en la concepción de Jesucristo. Aquí el texto griego

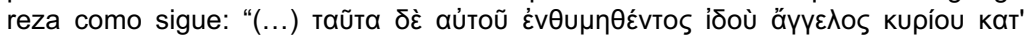

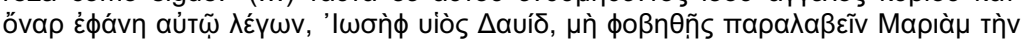
үuvaĩká бou, Tò үà viene del texto griego del Nuevo Testamento editado electrónicamente en el Thesaurus Indogermanischer Text- und Sprachmaterialien (T.I.T.U.S.), última consulta: $21.02 .2012<$ http://titus.uni-frankfurt.de/texte/etcs/grie/gnt/gnt.htm>). El texto griego utiliza yuvaĩká para referirse a María. Ahora bien, por desgracia, el comienzo del Evangelio de san Mateo no nos ha llegado traducido al gótico, por lo que esta incógnita tendrá que quedar sin despejar.

Futhark 8 (2013)

Ayerbe, Diferencias en la traducción, 9-30

ISSN 1886-9300 
función o categoría social (таıঠíokn), mas dicha distinción no se constata en la traducción gótica, ya que se utiliza tanto para una como para otras el término piwi.

Para barn y brups no hemos constatado muestras para hacer alusión a la Virgen María, sin embargo, ello no constituye indicio negativo de que ambos términos fueran incompatibles para ser empleados para referirse a ella. Es evidente que la Virgen María tuvo que haber sido niña en la primera etapa de su vida, igual que las demás mujeres. Lo único que ocurre es que los textos neotestamentarios no recogen su infancia y, en consecuencia, queda eliminada la posibilidad de hablar de ella como niña. En cuanto a brups, hay que decir también que, si bien no hemos hallado muestra alguna aludiendo a la Virgen María, tampoco lo hemos conseguido en el caso de otra mujer, salvo en el pasaje en el que, de modo genérico, Jesucristo profetiza que se rebelará la nuera contra su suegra ${ }^{13}$. Nuera fue también María por el hecho de estar casada con José.

Así pues, las distinciones constatadas se concentran en magaps

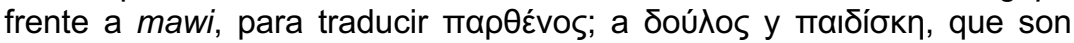
traducidos al gótico indistintamente con piwi; a la matización "desposada" en el uso de qens al referirse a la Virgen María, tanto en gótico como en griego. En los casos primero y último, proponemos como explicación, como ya adelantábamos anteriormente, la necesidad de resaltar la condición de perpetua virginidad de María, tanto antes como durante su matrimonio con José, así como antes, durante y después de dar a luz a Jesucristo. De no haber hecho estas distinciones, habría sido más difícil advertir al lector acerca de esta peculiar y exclusiva condición de María, pues es propio de cualquier mujer legítimamente casada cohabitar carnalmente con su marido, así como tener descendencia exclusivamente de él. De hecho, estas dos circunstancias entran dentro de los fines que Dios mismo había establecido desde el principio de la Creación para el matrimonio. En María, en cambio, no se dio ninguna de estas dos circunstancias (cohabitación carnal y concepción por obra de varón) y era necesario, por tanto, dejarlo claro de algún modo a nivel lingüístico.

Ahora bien, todo lo dicho hasta el momento, se refiere a las conclusiones del estudio exclusivamente en el ámbito de la lengua gótica. A un nivel contrastivo quedaría responder a la pregunta acerca de si los resultados constatados en gótico coinciden en alguna medida con los obtenidos ya en otras lenguas germánicas, que, en estos momentos, están limitados al alto alemán antiguo (Ayerbe 2011a) y medio (Ayerbe 2011b), y al sajón antiguo (Ayerbe 2012). No obstante, este propósito debe quedar, en nuestra modesta opinión, para un estudio posterior por

13 Cfr. Evangelio de san Mateo X, 35. Futhark 8 (2013)

Ayerbe, Diferencias en la traducción, 9-30

ISSN 1886-9300 
dos razones: la primera, porque haría falta antes contar con resultados en otras lenguas germánicas como el inglés antiguo y el nórdico antiguo. Ello permitiría partir de una visión más global que aportaría sin duda más elementos de comparación. En segundo lugar, porque los estudios realizados hasta la fecha aportan indicios de que el inventario de términos del campo léxico 'mujer' no es el mismo en todas las lenguas, por lo que habría que comenzar estableciendo en algunos casos qué términos equivalen a otros en la misma función y el mismo significado en cada una de las lenguas. $Y$ es esta una tarea que, aunque la consideramos necesaria, sobrepasa con creces el tema y el alcance del presente trabajo. Ésta habrá de ser nuestra tarea en futuros trabajos.

\section{Bibliografía}

AYERBE LINARES, Miguel, "El campo léxico 'Mujer' en sajón antiguo y su aplicación a la Virgen María: ¿igualdad o selección?", enRevista de Filología Alemana 20 (2012), (en prensa).

__ " ¿Hay diferencias en el uso de términos del campo léxico 'Mujer' en textos del alto alemán antiguo?", enEstudios Franco-Alemanes 3 (2011a): (en prensa).

_-, "Aportaciones de la literatura mariana medieval a la determinación léxica en la traducción del alto alemán medio: aplicación al campo léxico 'Mujer'”, en LÓPEZ Folgado, Vicente (ed.), Essays on Translation, Hamburgo, Dr. Kovač, 2011b. (en prensa).

BASTERO DE EleizALDE, Juan Luis,María, Madre del Redentor, Pamplona, EUNSA, 1995.

Bosco Coletsos, Maria Sandra, “'Dona' 'Moglie' nei Principali Dialetti Germanici Antichi”, enAevum 54:2 (1980), págs.257-279.

BRAUNE, Wilhelm, "Nhd. Braut in den Germanischen Sprachen“, en Beiträge zur Geschichte der Deutschen Sprache 32 (1907), págs.30-59.

FEIST, Sigmund, Vergleichendes Wörterbuch der Gotischen Sprache. 3., neu bearbeitete und vermehrte Auflage, Leiden, E.J. Brill, págs. 1939.

Novum Testamentum Graece, 09.12.2008, Thesaurus Indogermanischer Textund Sprachmaterialien de la Universidad de Fráncfort del Meno, 22 de febrero de 2012 <http://titus.uni-frankfurt.de/texte/etcs/grie/gnt/gnt.htm>.

SHIPP, Georg, 'Words for 'Man', 'Woman', and 'Wife' in some Indo-European Languages", en STEPHENS, Anthony (ed.),Festschrift for Ralph Farell, Fráncfort del Meno, Peter Lang, 1977, págs. 165-173.

STREITBERG, Wilhelm (ed), Die Gotische Bibel. Bd. 1: Der Gotische Text und seine Griechische Vorlage. Mit Einleitung, Lesarten und Quellennachweisen sowie den kleineren Denkmälern als Anhang. 7. Auflage, Heidelberg, Carl Winter, 2000. ${ }^{14}$

14 Incluye el texto del Skeireins, págs. 456-471, a modo de anexo.

Futhark 8 (2013)

Ayerbe, Diferencias en la traducción, 9-30

ISSN 1886-9300 